\title{
Study on the Virtual and Real Processing of Spatial Performance in Joseon Dynasty's Figure Paintings
}

\author{
Baoliang $\mathrm{Xu}$ \\ Xiamen Academy of Arts and Design, Fuzhou University \\ Xiamen, China
}

\begin{abstract}
The virtual and real performance treatment of Oriental painting has always been an important topic in the creation of Joseon Dynasty's Figure Painting. The split-joint relation of virtualness and reality, the performance of strength and weakness not only has the importance of determining the style, but also it has its own variability. The virtual and reality echoed with each other, their combination and mutual birth will form a complex and changeable relationship. By dealing with the various relationships between the virtualness and reality, the rhythm of the ups and downs is formed in the paintings, showing a certain sense of rhythm. Of course, the formation of this sense of rhythm is closely related to the virtualness and reality processing of the physical spatial performance of Joseon Dynasty's Figure Painting, the virtualness and reality processing of color spatial performance, and the virtualness and reality processing methods of pen and ink spatial performance. To a certain extent it expresses the aesthetic concept of Yin-Yang in Joseon Dynast's Figure Paintings.
\end{abstract}

Keywords-spatial performance; Joseon Dynasty's figure painting; virtualness and reality

\section{INTRODUCTION}

"All things are yin and yang", this is the necessary principle to deal with the performance of Joseon Dynasty's Figure Painting.

The theory of yin and yang is nothing but "virtual" and "real". In the expression processing of the artistic imagery of Joseon Dynasty's Figure Painting, the unity of contradiction between the virtual and the real has both its necessity and its importance. Only by dealing with the relationship between the two can we be born from nothing, be virtual from real, and be able to "virtual and spiritual, empty and wonderful"; it is also to be sure that real come from virtual, so that to achieve the perfect artistic conception of "The real cannot be too real, then the virtual will come itself'.

If the creation of Joseon Dynasty's Figure Painting is implemented in the practice of performance, the relationship between the virtual and the real, the strong and weak performance not only has the importance of determining the style, but also has its own variability. The combination of virtual and real will conflict and merge with the most direct ink, color and composition. If we can resolve the conflict and maintain the fusion of various relationships in the picture, we will clearly express the virtual and real unity in the figure painting. That is, the virtual and reality echoed with each other, their combination and mutual birth will form the rhythm of the ups and downs, showing a certain sense of rhythm. Of course, the formation of this sense of rhythm is closely related to the virtualness and reality processing of the physical spatial performance of Joseon Dynasty 's Figure Painting, the virtualness and reality processing of color spatial performance, and the virtualness and reality processing methods of pen and ink spatial performance. These three kinds of spatial processing methods are not only play an important role in the virtual and real processing in Joseon Dynasty's Figure Painting creation, and it is an effective analysis and specific interpretation of the virtual and real processing techniques. It is also an important academic extension factor to promote the development of Joseon Dynasty's Figure Painting.

\section{THE VIRTUAL AND REAL PROCESSING OF THE}

PHYSICAL SPATIAL PERFORMANCE IN JOSEON DYNASTY'S FIGURE PAINTING

Joseon Dynasty's Figure Painting has been extremely expressive since ancient times.

Although the aesthetics of the West is involved in the creation of oriental paintings and the re-exploration of regional culture, the expression of modern oriental figure paintings has expanded in depth and breadth. The rapid update of the appearance of oriental figure painting and the rapid development of figure painting techniques, the virtual space and performance processing are still the basic philosophical propositions that cannot be ignored.

Then, the first thing to deal with in the creation of figure painting is the virtual and real processing of physical space. Relatively speaking, the physical space is not only the physical properties of paper, brush, and ink that we generally understand, but has more enrichment and meaning. The most direct physical space for Joseon Dynasty's Figure Painting creation is the rich expression of the drawing shape on the screen, especially the visual depiction that appears on the composition, that is, the "real image". This "real" is definitely a big part of the basic form of the picture. The relationship is also the most realistic picture processing, that is, the layout said by ancients. The beauty of the layout lies in solving the conflicting relationship of the picture, because when a shape enters into the painting paper, various spatial relationships begin to grow, from one to two, from two to three, layers by layers without stop. So the first thing to ensure is the intuitive 
theme of the picture. The artistic image can be fully summarized, which is the main purpose of a figure painting.

To do this, in addition to using "real" to occupy the real space of the picture, it is necessary to think more on the "virtual". Because use virtual to contrast real could make the real part of the picture can be fully and prominently expressed. For example, in the 17th century Joseon Dynasty era painter Yin Douxu's "Self-portrait" in "Fig. 1", the most prominent part of the painting is the artist's own face, but not just a face, the painter needs to use the surrounding blanks to highlight the importance of the face. Therefore, there is no coloring on the choice of clothes, white clothing only carries a shallow hook line on the shoulders of the clothes. So on one hand, it implies that the fashion that scholars of the Joseon Dynasty are favored white color, they like to wear white clothes; on the other hand, it can stand out the specific shape of the figure's entire face. As is known to all, in the depiction of Oriental figure painting, especially the ordered works of some scholar-officials, most of them adopt the figures sitting or standing, and drawn from the front of the body, and will not like the Western tradition to adopt a side of $45^{\circ}$ in the structure of human body, and use the twisted and dynamic body muscles to describe the figures, so that the whole body and face have dynamic and light effects. The description of the positive facial expressions directly uses light to enter from the front, or even from all sides, the human body and face are easy to fall into the rigid stiffness, so that attention must be paid to the figure's facial expressions. These attentions are extremely subtle, for example, the fluctuations of facial muscles, the deep depiction of facial expressions, the choice and movement of facial wrinkles are all needed overall processing. This kind of processing has its own primary and secondary points, such as the conscious depiction of the eyelids and eyebrows, the angle of the eyebrows, the depth and subtleness of the pupils, and the portrayal of the muscles in the eyelids, it is easy to express the personality characteristics of the figures. Under the depiction of the calm brushstroke of his "Self-portrait", the painter Yin Douxu accurately grasped the physical structure of the facial contour and the depiction of the eyelids, pupils and eyebrows, which can clearly express his resolute character and the temperament of justice. Since these details are portrayed with very thin strokes, and the use of the waste line is abandoned, the expression is very accurate, then this requires a more virtual, lighter, and more simple part to set off the complex performance of the face, so when Yin Douxu made the depiction of the figure's clothes, he only outlined the contour, and the wrinkles of the clothes were basically unoutlined or less outlined.

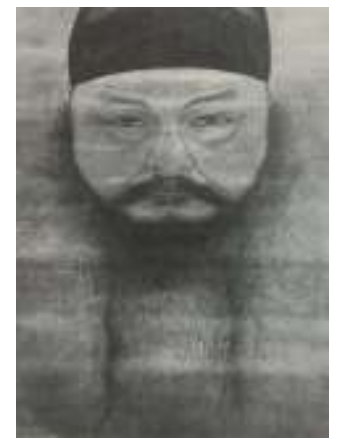

Fig. 1. Self-portrait of Yin Douxu.
In addition, based on the reality is the basic model in picture space is guaranteed, the background processing of Yin Douxu's "Self-portrait" in "Fig. 1" only used the original color of the paper for contrasting. In the painter's view, the depiction of the figure itself has already been infinitely unlimited, so there is no need to decorate the main part with any decoration, and no unnecessary background is needed to contrast, he carried out the depiction of the main part in the simplistic background.

Obviously it is because the virtual physical space that shows the real appearance.

This virtualness is also generated relative to reality. Therefore, "virtualness" at some point really depends on the success or failure of "reality". This requires that each spot dyeing will leave a gap for the next spot dyeing, leaving a thick, light, virtual, real strokes colliding, mutual generation, and mutual change in this space. Even under the thick pen and ink and structure, it will not be stuffy, revealing a little virtual white, like "one candle lights the whole room", forming a beauty with the virtual within the real.

Contrary to this, the hat of "Self-Portrait" is depicted in a real way. But the hat itself has its own structure, so there are two faint virtual whites at the top of the hat to indicate the corner of the hat. Although it is virtual white, but the hat is still very strong in terms of volume, and it is very simple without more changes and more physical depictions, which is different from the soft and complex muscles of the face. However, as far as hat is concerned, Yin Douxu undoubtedly used the method of seeking reality from virtualness, and he achieved a total effect of less is more and holds a part as the whole in artistic conception and virtual and real performance

It can be seen from the above analysis results that whether it is use virtual to contrast real, or use real to seek virtual, it will produce a certain prosody and rhythm in the physical space of the picture. As long as it is used properly, it is easy to clearly reflect the physical space virtual and real processing means in figure painting, these means play an important role in the spatial structure. These roles are hidden and can only be discerned with practice, thinking or analysis.

The method of virtual and real performance processing in physical space can open up a very broad space for creation itself, from physical space expression to artistic conception space, from artistic expressiveness to rich aesthetic connotation, the virtual and real performance processing are infinitely changeable and very effective. It is one of the important aesthetic expressions that cannot be ignored in Oriental painting.

\section{THE VIRTUAL AND REAL PROCESSING OF THE COLOR} SPACE EXPRESSION OF JOSEON DYNASTY'S FIGURE PAINTING

The Oriental traditional figure painting is the best at grasping the relationship between virtual and real. Whether it is the "Nv Shi Jian Tu" or the "Dao Lian Tu", the essence lies in the organic cooperation between virtual and real, especially the mutual reflection and mutual integration in colors, which make the whole picture have a unique appearance, fully reflect the artistic tension of "mutual generation of virtualness and 
reality", and thus maximize the potential effectiveness of the two aspects of virtual and real processing in figure painting. That is to say, there is a mutual interaction between virtual and real. When necessary, "real" gives "virtual" powerful artistic life, and "virtual" brings "real" full artistic spirituality.

The virtual and real of the color space of Joseon Dynasty's figure painting is often reflected in the density of the image and the change of color. Paying attention to reality and pursuing virtualness is also a place where the color performance is different.

There are several different forms of color space change in Joseon Dynasty's figure painting creation:

First of all, use real to express virtual. The typical color space performance should be the 17th century Joseon Dynasty painter Li Mingyu, whose representative work is "Yu Qiao Wen Da Tu" in "Fig. 2". This painting depicts the scene of the two mountain people who live seclusion in the mountains, the joy and leisure between the fisherman and the woodman's speech, and the calmness and smoothness between the eyebrows fully express the unrequited joy of mountain life, which is the topic that officials in Joseon Dynasty were happy to discuss. Of course, the main part of the painting is the fisherman and the woodman, and reveals the theme through their conversations and actions. However, in the real scene of the painting, Li Mingyu still concentrated and elaborated on the large area of bamboo, even it is the strongest ink part in the painting. In the middle of the strong ink color, Li Mingyu not only used the light ink to set off the surrounding of the bamboo, but also consciously added cyanine and mineral green in the ink color, which added the color structure change to the bamboo part. According to common sense, the excessive detailing of the non-subjective part and the variability of the color structure will have a negative effect to steal the show of the whole painting.

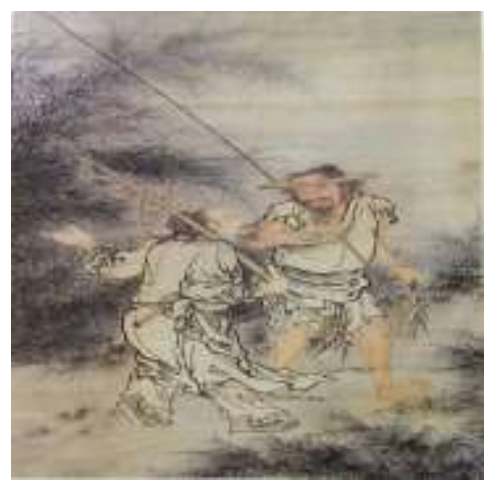

Fig. 2. Li Mingyu's "Yu qiao wen da tu".

However, Li Mingyu's depiction of bamboo seems to be something else. The reason why use a variety of color space structures to draw bamboo is to deliberately set off the character structure of the conversation between fishermen and woodman, use strong to contrast the light, use real to contrast the virtual, the two figures light and fluttering image has been contrasted out. Then, the relatively light color space of the fisherman and the woodman becomes the main part of the virtual and real contrast. Even the main part can express its wonderful part without over-expressing the color. The exciting part is not the color display, but to use color to set off the main body, so the line expression on the main body is more clear and wonderful.

Second, use virtualness to bring reality. The color space with virtual and real can still find very good performance in $\mathrm{Li}$ Mingyu's "Yu Qiao Wen Da Tu" in "Fig. 2". However, Li Mingyu's real and virtual approach is not easy to detect, and it is very easy to use the virtual color space to deal with the clear expression of the nature of the object.

The most obvious and wonderful one is the outline of rocks and color use in the painting. He first outlined two strokes of earth and stone in the lower part of the picture, and only made the outline of the earth and stone structure between the fisherman's legs, and it was very inconspicuous. After that, these few earth and stone lines were used as the base for extension, light cyanine was used to slightly dye along the direction of the earth-rock line, and the dyeing was very incomplete. Only dyed the specific direction of the earth and stone and it was very blurred, thus forming the illusion as if there is nothing, and then rubbing two strokes in the back part of the woodman's feet as a wake-up stroke of the virtual part of the color space, so that the spatial structure of the earth-rock road is more obvious, and the homeopathy has a dynamic sense. That is to say, the virtualness of the color space is not only limited to the reality of the color space, but also the virtual and real of the brush stroke. Which means the common virtual and real performance of color and the hook line and the rubbing can easily complete the depiction of the earth and stone slope. The earth-rock slopes thus displayed not only have soft and loose characteristics, but also match the dynamics of the human body. With a very soft, looming coloring structure, it achieves an unexpected effect, fully demonstrating the unique charm of the virtual and real color space processing method.

Moreover, virtual used as real, and real is expressed with virtual.

This is the most difficult method to use virtual and real processing in color structure, and it is also the most effective way to make the plain picture perfect. Of course, it is not enough to only use virtual and real processing method in a painting. More common method is the combined use of multiple virtual and real techniques. Therefore, in a painting, virtual used as real, and real is expressed with virtual may be combined, especially in some classic works. For example, Li Mingyu's "Yu Qiao Wen Da Tu" in "Fig. 2".

In the Qing Dynasty, Fang Xun said that: "The dark and light of color set is not difficult, it is the color match that is difficult". To deal with the overall virtual and real relationship in the color space of the picture, we must not only arrange the relationship between the large virtual and real and small virtual and real, the actual change of the virtual and real must start from the specific each stroke, and also it must control from the overall structure of the picture. In addition to the depiction of the main characters in the "Yu Qiao Wen Da Tu", the most widely occupied space is the virtual drawing of the sky part, which is more singular than the overall part. However, it is contrasting with the changing color space of the main part, and creating a vast sky with a cloud-like atmosphere seems to be 
the conversation content of the fisherman and woodman. The background of the whole picture is highlighted by the invisible virtual color. Although the monochromaticity of the cloud is virtual, the vast sky is real, and it can even lead to the purpose of the conversation of the figures.

In the contrast between dynamic and static, virtual and real, the texture is real, and the real is expressed with virtual, and it has a dynamic sense of rhythm; the application of color is loose and spiritual, it is more like use virtual to express real, and the depiction of bamboo color is the like this. Throughout the painting, the actual painted bamboo is only two to three clumps, but it is still use the contrast of ink and light-colored cyanine, and even directly use color to make bamboo, which further creates the density and depth of the bamboo forest, and suddenly the whole picture is full of vigour, but the space of imagination it left has expanded infinitely. This undoubtedly adds a sense of glamour in the relatively dull color space structure, the echo between virtual and real could effectively express the characteristics of each object, and reflect subtle level changes in the color space.

From the above point of view, the virtual and real performance processing of the color space in Joseon Dynasty's figure painting deals with the contradictory relationship of the picture in the duality of the reality is virtualness, virtualness brings reality, virtualness uses as reality, and reality expressed with virtualness. From virtual to real, from real to virtual, even to shady, light and heavy can be all be processed through the virtual and real performance of the color space, and finally complete the control and improvement of the whole picture in the organic cooperation of virtual and real.

\section{The Virtual and Real Processing of InK SPaCe PERFORMANCE IN JOSEON DYNASTY'S FIGURE PAINTING}

\section{Every Oriental painting must use pen and ink.}

With the turbidity and distinctness of the pen, the multicolored changes of the ink, the contrast between virtual and real, and the subtle creation, the virtual and real processing of the pen and ink space has been pushed to the extreme, and it has become one of the typical signs of Joseon Dynasty's painting creation.

The pen and ink space of Joseon Dynasty's figure painting is difficult to separate from the processing of virtual and real, yin and yang. Although it is a commonplace, it is the most versatile part of the creation of figure painting. The virtual and real, clever and ambiguous, rigid and soft, thick and thin, square and round, curved and straight that expressed in the pen and ink space are always the opposite elements of the painting, and the virtual and real of the pen and ink space becomes the generality to deal with various opposites.

The importance of the generality is that one piece of white paper, once pen and ink enter the white space, yin and yang are generated.

The spatial cutting of the ink line on the paper will lead the change on layout distribution. Even if it is one ink line, one ink dot, the virtual and real relationship on the white paper will be ever-changing, and the pure "white paper" will no longer exist.
When the pen and ink enter the white paper to create the pen and ink space, the pen method and spatial distribution become an important step in dealing with the transformation of the virtual and real relationship, especially for the Joseon Dynasty's figure painting. The scorching, shading, backing, and transfer of pen use is an important way to express the brushwork, and with the application of water in the ink pen, such lines are undoubtedly rich in expressiveness, whether in their own line structure or in terms of emotional expression, the method of pen use is the most attractive part of Joseon Dynasty's figure painting. In the Qing Dynasty, Hualin said in "Nan Zong Jue Mi" that: "It is beyond pen and ink use. It can make the painting not only has white paper, but also with sentimental, otherwise the painting is not alive". So the change and expressions of the pen use, whether it is a solid line, or a dotted line, or a crossing with dotted and solid line, or a line with virtual and real in it will all make the picture vivid and far-reaching.

Therefore, the pen use of Joseon Dynasty's figure paintings can be described as nothing without virtual and real.

Sparse and dense, loose and tight, light and heavy, thick and thin, complex and simple, guest and master, press and expose become the external extension of the virtual and real. Among them, sparse, loose, light, thin, simple, guest, and press are embodied as "virtual", dense, tight, heavy, thick, complex, master, expose are embodied as "real". In the Ming Dynasty, Tang Zhiqi talked about the pen use in "Hui Shi Wei Yan" that:"There are more hiding than the expose, and the fun is more and endless", this fun is the hide and expose exchange in pen use, when the characters are depicted, the use of pen has both a practical part and a virtual part. Contrast between reality and virtualness, it is easy to highlight the essential characteristics of things, so as to ignore other non-essential things. Even if there is no ink, you can interact with the ink and generate each other to express the central theme of the picture.

In addition, the pen and ink space in Joseon Dynasty's figure painting itself has the artistic characteristics of hypothesis and virtuality.

It is this hypothesis and virtuality that make the picture itself have the assumption of virtual and real image, not just the reproduction of the real image, but it is the part of the figure painting creation that is different from the Western painting. Joseon Dynasty's figure painting valued virtual spirits, focus on connotation, know how to use the virtual, so that pen and ink space can be extended infinitely, and the artistic concept that can be expressed is more intriguing.

The creation of Joseon Dynasty's figure paintings also naturally divides ink into five colors, especially in the creation of freehand figure paintings. In fact, the five colors of ink are only in general terms, in actual practice, the subtle differences in ink color are more than thousands. Generally speaking, the strong contrast of ink performance is not difficult, and it is difficult to have both strong contrast and also subtle changes. It is necessary not only to have a strong and harmonious contrast with virtual and real and rhythm, but also to have a clear overall sense under the control of virtual and real. 
The ink method has various changes, but it has a commonality with the pen method. It aims to create the artistic conception that can be expressed by the ink and water law with virtual and real processing. In the creation process of figure painting, ink method like landscape, bird and flower paintings, it has to make many ink and water elements, such as virtual and real, hide and expose, clear and turbid, gather and scatter, straight and curve, open and close, strong and weak, dry and wet, sparse and dense, loose and tight, link and broken, complete and apart, and other the binary contradictions, to handle all kinds of hedging relations perfectly, and make overall arrangements according to the overall creative intention.

For example, the Korean era painter Li Cheng's "Fang He Tu" in "Fig. 3", for the hermit's clothing, the brushwork can be said to be strong and very positive. Such a solid brushwork, on one hand, is to form a unity with the stone that the hermit sits down, on the other hand, it adds to the sense of stability of the hermit sitting on the rock. The most important thing is that the realistic line of hard clothing pattern is just like the line of the hermit's face, especially the line of the beard is softly fluttering, while at the same time ensuring a certain shape, the brushwork of beard is very elegant. This is undoubtedly a turning point in the use of pens and inks, or a contrasting part. Li Cheng combines the virtual and real with the rhythmic changes of the pen and ink method. In this way, the clothing pattern is real and the skin and the beard of the hermit are virtual, and the inklessness between the pens is also virtual. After the combination of virtual and real, even the virtual blank space that does not have the ink will coincide with its own virtualness and reality. Or even if it is a flat ink, it has its own virtual and real level. It is called "virtualness makes all landscapes in", and "virtualness" is where the "reality" hided, so it contains an infinite sense of rhythm. This kind of virtual rhythm can give people the beauty that is beyond the reach outside the object.

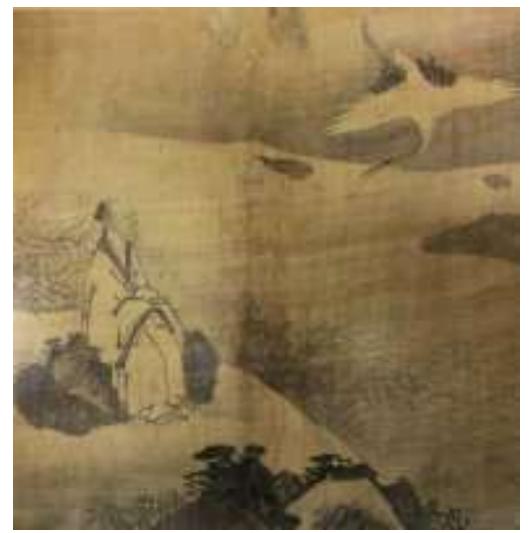

Fig. 3. Li Cheng's "Fang he tu".

In addition, the protagonist of "Fang $\mathrm{He}$ Tu" did not use a fine pen to outline, but in the process of virtual and real processing of ink and pen use, the clever transition of figure paintings has been successfully completed, so that to achieve the unity and opposition between the virtual and the real. Just as in "Feng Yu Gui Zhou Tu", Shen Zhou used the ink method and virtual and real method to deal with the characters and the surrounding environment, the far sand was wiped out, the rain was overwhelming, the boat was alone and the wind and rain were mixed, and the virtual and real processing method of the pen and ink space were used to create the artistic conception that invisible is better than visible and visible can create invisible. This has produced unimaginable effects on the breadth and depth of the aesthetic creation in Joseon Dynasty's figure painting.

\section{CONCLUSION}

The relationship processing between virtual and real of Joseon Dynasty's figure painting is particularly important.

The virtualness and reality not only have rich connotation in the creation of Joseon Dynasty's figure painting, but also an important means of shaping its meaning beyond words. It is a powerful interpretation of the implicit beauty of oriental traditional culture. In the creation of Joseon Dynasty's figure paintings, the method of real and virtual payout, seek virtual with real, seek real with virtual, and communicating with each other has variability. This kind of variability, whether in physical space processing, color space processing, or in the pen and ink space processing, all show a certain contradiction and richness. Especially the change process when dealing various conflicts and contradictions with virtual and real processing, and the role played in the process of change is the charm of Joseon Dynasty's figure painting.

Specifically, in the case of direct practice creation, the relationship between virtualness and reality, the strong and weak performance will conflict and contradict with the most direct ink, color and composition. The solution of conflicts and contradictions, the integration of easing various opposing relations, using the method of virtual and real echoed with each other to create a certain picture rhythm and shows a certain sense of rhythm. Of course, this sense of rhythm can achieve perfect unity through the virtual and real performance processing of the physical space, the virtual and real performance processing of the color space, and the performance processing of the pen and ink. The virtual and real processing methods of these three spatial expressions not only played an important role in the creation of Joseon Dynasty's figure paintings, but also showed the aesthetic concept of Joseon Dynasty's figure paintings. Therefore, the virtual and real relationship processing in the paintings of Joseon Dynasty is an important issue which is difficult to avoid. It is also a practical way to continue the development of Joseon Dynasty's figure paintings, furthermore it is an important aesthetic basis for Oriental art creation.

\section{REFERENCES}

[1] Jiang Kuanzhi (Korea). Jian song wen hua.The visual realism of the painter's paintings in the late Real Scene Era [M]. Korea National Academy of Fine Arts, 1995. 姜寬植（韩）.澗松文華.真景時代後期 畫員畫的視覺的寫實性 [M].韓國民族美術研究所, 1995 .

[2] Xiao Xiaoi. Kong na wan jing__On the "Blanks" and Joseon Dynasty Painting [D]. Northeast Normal University, 2010. 肖霄. 空纳 万境一一试论“留白”与李朝画[D].东北师范大学, 2010 .

[3] Huang Ying. Meaningful with Invisible shape, voice with silence [D]. Nanjing Normal University, 2005. 黄娈. 无形有意无语有声[D]. 南京 师范大学, 2005 . 
[4] Jiang Liangfu. Interpretation of Laozi and Zhuangzi [M]. Hangzhou: Zhejiang Literature and Art Publishing Company, 1994. 姜亮夫. 老子 庄子直解[M]. 杭州: 浙江文艺出版社, 1994 .

[5] Han Linde. Research on Shi Tao and painting quotations [M]. Nanjing: Jiangsu People's Fine Arts Publishing Company, 1996. 韩林德. 石涛 与画语录研究[M]. 南京: 江苏人民美术出版社, 1996.

[6] Pu Zhangai (Korea) Real Scene Landscape Painting [M] Seoul: Sungkyunkwan Publishing Department, 2014. 朴章爱(韩) 实景山水画 [M]首尔: 成均馆出版部,2014.

[7] Wang Bomin. Huang Binhong's painting quotation [M]. Shanghai: Shanghai People's Fine Arts Publishing Company. 1997. 王伯敏. 黄宾 虹画语录[M]. 上海: 上海人民美术出版社。1997.

[8] Zhu Pu. Lin Fengmian's painting language - Ming shan hua tan [M]. Shanghai: Shanghai Fine Arts Publishing Company, 1997. 朱朴. 林风 眠画语——明山画谭[M]. 上海: 上海美术出版社, 1997. 